\title{
European Isokinetic Society Congress
}

\author{
17-18 March 2000
}

Brugge, Belgium 


\section{EIS Show Schedule}

\section{Friday 17 March 2000}

13:00 Introduction M. Genty, France

13:15 Force Measurement in Research: State of the Art D. Schmidtbleicher, Germany

13:45 New Developments in the Medico-Legal Applications of Isokinetics Z. Dvir, Israel

14:15 Break

14:45 History of Isokinetics B. Herbeck, Germany

15:05 Use of Eccentrics in Assessment and Rehabilitation: A Critical Analysis J.L. Croisier, Belgium

15:35 Diagnostics with Isokinetic Devices in Shoulder Measurements: Potentials and Limits F. Mayer, Germany

16:05 Discussion

16:35 Break

16:55 Isokinetics and Low Back Pain: Principles of an Objective Evaluation and of the Rehabilitation Program J. Vanvelcenaher, France

17:15 Low Back Problems and Return to Work: Isokinetics?

R. Melcherts, The Netherlands

17:35 Isokinetic Assessment in School Children with Low Back Pain

S. Negrini, Italy

17:55 Discussion

18:10 End of the day

\section{Saturday 18 March 2000}

9:00 Isokinetics and EMG: Standardized Measure for the Lower Extremity

P. Konrad, Germany

9:30 Maximal and Sub-Maximal Torque-Velocity Relationships for Human Knee and Ankle Extensor Muscles

A. Thorstensson, Sweden

9:50 The Use of Isokinetics in Treatment of Patellofemoral Problems

U. Geiger, Switzerland

10:10 Discussion

10:30 Break

\section{1:00 Workshops}

Poster sessions: F. Mayer, Germany

Workshop 1: Back Testing and Functional Restoration Speaker: P. Voisin, P. Bibre, France Equipment: CYBEX

Workshop 2: EMG \& Isokinetics Speaker: P. Konrad, M. Dahlinger, Germany Equipment: Noraxon \& BIODEX

Workshop 3: Shoulder Speaker: B. Herbeck, Germany Equipment: BIODEX

Workshop 4: Clinical Aspects of Eccentrics in Isokinetic Application Speaker: B. Forthomme, J.L. Croisier, J.D. Moureau, Belgium Equipment: CYBEX

12:00 Workshops

Poster sessions: F. Mayer, Germany

Workshop 1: Back Testing and Functional Restoration Speaker: P. Voisin, P. Bibre, France Equipment: CYBEX

Workshop 2: EMG \& Isokinetics Speaker: P. Konrad, M. Dahlinger, Germany Equipment: Noraxon \& BIODEX

Workshop 3: Shoulder Speaker: B. Herbeck, Germany Equipment: BIODEX

Workshop 4: Clinical Aspects of Eccentrics in Isokinetic Application Speaker: B. Forthomme, J.L. Croisier, Equipment: CYBEX

13:00 Lunch in Exhibition Area

14:00 Workshops

Poster sessions: F. Mayer, Germany

Workshop 1: Back Testing and Functional Restoration Speaker: P. Voisin, P. Bibre, France Equipment: CYBEX

Workshop 2: EMG \& Isokinetics Speaker: P. Konrad, M. Dahlinger, Germany Equipment: Noraxon \& BIODEX

Workshop 3: Shoulder Speaker: B. Herbeck, Germany Equipment: BIODEX 
Workshop 4: Clinical Aspects of Eccentrics in Isokinetic Application

Speaker: B. Forthomme, J.L. Croisier, J.D. Moureau, Belgium

Equipment: CYBEX

15:00 Break

15:20 Workshops

Poster sessions: F. Mayer, Germany

Workshop 1: Back Testing and Functional Restoration Speaker: P. Voisin, P. Bibre, France Equipment: CYBEX

Workshop 2: EMG \& Isokinetics Speaker: P. Konrad, M. Dahlinger, Germany Equipment: Noraxon \& BIODEX

Workshop 3: Shoulder Speaker: B. Herbeck, Germany
Equipment: BIODEX

Workshop 4: Clinical Aspects of Eccentrics in Isokinetic Application Speaker: B. Forthomme, J.L. Croisier, J.D. Moureau, Belgium

Equipment: CYBEX

16:30 Panel Discussion and Closing Remarks D. Schmidtbleicher, Germany

17:00 End

\section{Chairman}

Friday afternoon: J.L. Croisier

Saturday morning: M. Genty

Saturday afternoon closing session: D. Schmidtbleicher 


\section{List of Speakers}

Mr. Frank Mayer, M.D.

Eberhard-Karls University Tübingen

Medical Clinic, Department Sports Medicine and

Clinic of Orthopaedic Surgery

Hölderlinstrasse 11

D-72074 Tübingen

Germany

Mr. Regi Melcherts, M.Sc. PT

OCA institute The Netherlands

Maanlander 10

3824 MP Amersfoort

The Netherlands

\section{Mr. Bernd Herbeck, PT}

Sportomed Reha GMBH

Im Pfeifferswörth 4

D-68167 Mannheim

Germany

Mr. Jacques Vanvelcenaher, M.D.

Medical Director

Centre de Rééducation Fonctionelle L'Espoir

25 Pavé du Moulins

59260 Lille Hellemmes

France

\section{Mr. Urs Geiger, PT}

GYM medico

Hebelstrasse 56

4056 Basel

Switzerland

Mr. Jean Louis Croiser, PT, PhD

University of Liege

Department of Physical Therapy and Rehabilitation

CHU Sart Tilman B35

4000 Liege

Belgium

\section{Mr. Peter Konrad, PhD}

KinLab - University of Dortmund

Otto-Hahn strasse 3

44227 Dortmund

Germany

Prof. Alf Thorstensson, PhD

Department of Sports and Health Sciences
Stockholm University College of Physical Education and Sports

Department of Neuroscience, Karolinska Institute

Box 5626

S-11486 Stockholm

Sweden

\section{Prof. Dietmar Schmidtbleicher, PhD}

Head and Chair Department of Movement and Training Science

Institute of Sport Sciences

J.W. Goethe University Frankfurt

Ginnheimer Landstrasse 39

D-60487 Frankfurt/M

Germany

Mr. Stefano Negrini, M.D.

Rehabilitation Department

Don Gnocchi Foundation, Care \& Research Institute

Via Capecelatro 66

20148 Milan

Italy

Prof. Zeevi Dvir, PhD, LLB

Sackler Faculty of Medicine

Tel Aviv University

P.O. Box 39040

Ramat Aviv, 69978 Tel Aviv

Israel

Mr. Philip Voisin, PT

Centre de Rééducation Fonctionelle L'Espoir

25 Pavé du Moulins

59260 Lille Hellemmes

France

Mrs. Bénédicte Forthomme, PT

University of Liege

Department of Physical Therapy and Rehabilitation

CHU Sart Tilman B35

4000 Liege

Belgium

Mr. Marc Genty, M.D.

CRF La Roseraie

7, Rue Charles Dalencour

76310 Saint Adresse

France 
Speaker Abstracts 



\title{
Force Measurement in Research: State of the Art
}

\author{
Dietmar Schmidtbleicher \\ Institute of Sport Sciences, J.W. Goethe-University Frankfurt, Germany
}

Posture and movements in humans are a result of strength, produced by the muscles and conducted by tendons, ligaments and bones, and by the interaction with the external conditions outside of our body. This interaction can be measured as external forces in a physical sense.

We use for example force transducers inside the equipment, i.e. shoes, skies, poles etc. — or we registrar the interaction between man and surface with force plates or pressure soles inside the shoes. The problem with this type of measurements consists in the fact, that we cannot decide which muscles are responsible for the produced forces.

Following the structural analysis of motor strength, we find beside the above shown "sports-specific strength" a more generally one, that is due to all type of posture and movement in sports as well as in daily life. This "basic strength" consists of; maximal strength (MS) and explosive strength (rate of force development, RFD) in concentric, isometric and eccentric muscle actions. In movements with a combination of eccentric and concentric components, we distinct additionally between a fast and a slow stretch shortening-cycle (SSC).

The diagnosis of basic strength qualities (MS, RFD) can be done with measurement devices for multijoint (translatoric, close chain) movements or for single joint (rotatoric, open chain) movements. Both types have advantages and disadvantages and are not comparable, and for both types exist a strong dependence between muscle length and force production. The variation of the external resistance with respect to the changing muscle length in a single joint movement shows the highest correlation between externally measured force with the internally produced strength and these are the basics of isokinetic measurements. 


\title{
New Developments in the Medico-Legal Application of Isokinetic Dynamometry
}

\author{
Zeevi Dvir \\ Sackler Faculty of Medicine, Tel Aviv University, Israel
}

Quantification of muscle performance is a critical component in the determination of muscle-related impairment and disability. As outlined in several studies, manual muscle testing cannot fulfil this role as one single grade: 4 , covers about $90 \%$ of muscular capacity. On the other hand, isokinetic systems are particularly suitable for this purpose. However, the assumption that patients are really performing at their maximal torque output, known by the term effort, is not one that should be taken for granted. In fact, only upon confirmation of a maximal effort level can the clinician proceed to the next phase namely determination of impairment.

The traditional approach for assessing the sincerity of effort has been to look for the consistency of the strength scores/curves. This approach has been shown to be erroneous due to an unacceptable rate of false negatives and positives. The alternative approach, developed by the present authors in 1995, was to look for extreme deviations in the index: $\mathrm{DEC}=(\mathrm{Ecc} / \mathrm{Con}) \mathrm{high}-(\mathrm{Ecc} / \mathrm{Con})$ low, where Ecc, Con, high and low refer to Eccentric and Concentric strength at high and low testing velocity. Statistical application of this index yields a clear answer in terms of the levels of confidence set. Its efficiency has been demonstrated in a number of studies relating to knee and trunk extensors, elbow flexors and handgrip.

Recently, sincerity of effort was reassessed with respect to trunk extensors using extremely short ROM and velocity gradient of 1:4 (low : high), during 2 test occasions at least a week apart. It was revealed that the DEC was between $90-100 \%$ efficient for identifying sub-maximal effort in men and about $75 \%$ in women. The implications of these findings are discussed. 


\title{
History of Isokinetics
}

\author{
Bernd Herbeck \\ Sportomed Reha GMBH, Mannheim, Germany
}

Based on the ultimate goal of measuring dynamic performance of human muscles, the isokinetic dynamometers were originally developed to quantify muscular output.

For the external dynamic measurement of the parameters "force/torque" and the link to the internally produced "strength" of human muscle it took until the late 1970's for the technical possibilities to be realised.

The force measurement of humans required safety features, which were accomplished by the isokinetic dynamometers, as long as healthy populations were involved, but it needed further adaptations for the clinical application with patients. This conflict and its denial of the resulting problems faced the isokinetic devices (apart of their approval) with serious difficulties; especially by insufficiently informed physicians and therapists.

Due to more refined thinking processes about neuromuscular function and the development in the field of exercise physiology, the integration of isokinetic systems in rehabilitation as a training and testing tool greatly advanced.

In today's use of the words "safety and accommodating resistance" they will be directly associated to the field of isokinetics. By the inherent nature of current isokinetic technology, resistance is performed on a higher level of accuracy compared to humans trying to do the same. Consequently the isokinetic dynamometers could be incorporated in professional rehabilitation strategies whenever adequate resistance is the selected method to improve function. If known limitations in the application are respected, they are outweighed by advantages, which cannot be achieved otherwise.

In the world wide limitation of financial resources isokinetic systems make an indispensable contribution to optimise evidence- based treatment procedures and to document efficiency now and in the future. 


\title{
Use of Eccentrics in Assessment and Rehabilitation: A Critical Analysis
}

\author{
Jean-Louis Croiser \\ Department of Physical Therapy and Rehabilitation, University of Liege, Belgium
}

Isokinetic assessment contributes to recent achievements in treatment and prevention of various pathologies. Some studies demonstrate the interest and discriminating qualities of eccentric evaluative trials:

- Isokinetic evaluation of hamstring strains [1] revealed a more important reduction of flexors peak torque during eccentric contractions ( $>20 \%$ on average) than for the concentric mode $(10 \%)$. A mixed ratio $\left(\mathrm{H}_{\mathrm{ecc}} / \mathrm{Q}_{\text {conc }}\right)$ appeared significantly reduced $(0,75 \pm 0,23)$ for the injured muscles in comparison with the contra-lateral non-injured $\operatorname{limb}(0,90 \pm 0,16)$ and with a control population $(0,98 \pm 0,14)$. Opposite, classical concentric $\left(\mathrm{H}_{\text {cond }} / \mathrm{Q}_{\text {conc }}\right)$ ratios didn't distinguish the strained muscles from the normal thigh.

- In the context of anterior cruciate ligament reconstruction surgery [2], a preferential reduction of eccentric torque values was also observed for the knee flexors.

Eccentric exercises allow original reeducative applications. According to the principle described by Stanish et al. [3], we have used isokinetic protocols for the treatment of chronic tendinosis, with a progressive increase of angular velocity and contraction intensity [4]. However, a critical analysis questions on the excessive or inadequate use of eccentrics:

- The high tensions generated through eccentric contractions raise the risk of instantaneous injury.

- Exercise-induced muscle damage has been widely reported after eccentric session of training. It can be felt as muscle soreness or pain a few days after exercise. Increases of myocellular protein levels in serum are used as indicators of muscle damage: creatine kinase sometimes reaches the high level of $10.000 \mathrm{UI} / 1$ [5]. A specific training permits to reduce the intensity of DOMS [6].

- To ensure an accurate isokinetic assessment, the following remarks have to be considered:

- Although remaining within acceptable limits, eccentric measurements appear less reproducible than concentric modality [7].

- During eccentric contraction, peak torque appears at the end of the movement, in a lengthening position. For this reason, an appropriate test protocol would reject highest eccentric velocities (superior to $120 \%$ ), to avoid a measurement in a non-isokinetic part of the curve [8].

\section{References}

[1] J.L. Croisier and J.M. Crielaard, Isokinetic Evaluation of Hamstring Muscle Strain: Interest of the Eccentric Mode, Proceedings of the Third Annual Congress of the European College of Sport Science, Manchester, England, 1998, p. 255.

[2] J.L. Croisier, J.P. Huskin and J.M. Crielaard, Isokinetic Evaluation of ACL Reconstruction. Surgical Procedure Influence Rehabilitation Implications, Proceedings of the 9th Congress of the European Orthopeadic Research Society, Brussels, 1999 , p. 073.

[3] W. Stanish, R. Rubinovich and S. Curwin, Eccentric exercise in chronic tendinitis, Clin Orthop 208 (1986), 65-68.

[4] J.L. Croisier, B. Godon, F. Giordano, B. Forthomme, M. Namurois and J.M. Crielaard, Tendinites rotuliennes et achilléennes: intérêt des exercices isocinétiques excentriques. In: Progrès en Médecine Physique et de Réadaptation (3ème série), Masson, Paris, 1999, pp. 212-217. 
[5] J.L. Croisier, G. Camus, T. Monfils, G. Deby-Dupont, M. Fafchamps, I. Venneman, J.M. Crielaard, A. Juchmes-Ferir, C. Lhermerout, M. Lamy and C. Deby, Piroxicam fails to reduce myocellular enzyme leakage and delayed onset muscle soreness induced by isokinetic eccentric exercise, Mediators of Inflammation 5 (1996), 230-234.

[6] J.L. Croisier, G. Camus, I. Venneman, G. Deby-Dupont, A. Juchmes-Ferir, M. Lamy, J.M. Crielaard and J. Duchateau, Effects of training on exercise - induced muscle damage and interleukin 6 production, Muscle Nerve 22(2) (1999), $208-212$.

[7] J.L. Croisier and J.M. Crielaard, Exploration isocinétique: analyse des paramètres chiffrés, Ann Réadaptation Méd Phys 42 (1999), 538-545.

[8] J.L. Croisier, Contribution fondamentale et clinique à l'exploration musculaire isocinétique, Thèse de Doctorat, Université de Liège, 1996. 


\title{
Diagnostics with Isokinetic Devices in Shoulder Measurements: Potentials and Limits
}

\author{
Frank Mayer and H.H. Dickhuth \\ Medical Clinic, Deptartment of Sports Medicine, Clinic of Orthopedic Surgery, Eberhard-Karls \\ University of Tübingen, Germany
}

The correction of "muscular dysbalances" is assumed to be important in therapy of various shoulder pathologies and muscular strengthening. However, it is not clear how these dysbalances should be defined and the extent to which conclusions can be drawn, which are relevant to therapy and training.

Statements on physiological strength ratios and deviations from these are only possible in valid and reliable recording. Thus isokinetic strength measurements can reliably differentiate up to $180 \%$ s between the various types of work and movements within test-retest variability of $15-20 \%$. The maximum torque serves as a reliable parameter, additional information is obtained by determination of local muscle fatigue.

It is presently assumed that age and sex-specific strength ratios can be reliably measured. Decisive is consideration of the test method and especially positioning and fixation. However, the direct application of isokinetically determined strength values in training and rehabilitation is only possible when the exercise position agrees with the position in measuring. This is usually not the case. Since isokinetic movements are almost not encountered in everyday living or sports, data obtained by these methods can hardly be used for training management and should mainly be used as a therapy control.

Adequately reliable data on the strength ratio of shoulder movements can be obtained within narrow limits by isokinetic strength measurements. The satisfactory standardisation and differentiation possibilities are one advantage. Additional stress in everyday living and sports lead to specific changes, which however should always be interpreted on the basis of method-specific criteria and exercise-specific movements. In spite of critical assessment of the methods, it is recommended that isokinetic strength measurements should be uniformly performed by query in order to guarantee comparability and interpretation. Taking functional-anatomical and methodical considerations into account, isokinetic strength measurements deliver valuable information for training and therapy within the framework of medically and training- scientifically founded strength diagnostics. 


\title{
Isokinetics and Chronicle Low Back Pain: Principles of an Objective Evaluation and of the Rehabilitation Program
}

\author{
Jacques Vanvelcenaher \\ Centre L'Espoir, Lille Hellemmes, France
}

Ninety percent of acute low back pain patients recover during the first three months whatever the treatment [6]. Ten percent go on to chronically. The treatment focuses on pain, with poor efficiency.

Until now, a data was missing: the deconditioning syndrome $[5,7,12]$. This syndrome brings out the physical consequences of chronic low back pain, whereas the trunk musculo-joint unit is the most important and the strongest of the total body biomechanical chain [13]. Psychosocial deconditioning is associated to physical disabilities [1]. But, what is needed is a quantitative technique for objectively documenting trunk function. To perform this original physical approach, we need some specific testing tools $[11,16]$.

Isokinetic devices gives specifics, objectives and normative data, for assess the physical impact of low back pain [2]. The benefits of isokinetics device are known; this is the most efficient use of muscle out put, it reduces the risk of overload injury, adapts to pain and fatigue and gives feed-back for patients and therapists. There is a virtual diminution of ballistic movement and an optimisation of the co-ordination.

Whatever the speed is, normal trunk extension curves are higher than flexion ones [14]. In the chronic low back pain patients group, isokinetic extension is $50 \%$ weaker $[3,16]$.

With dysfunction of the spine, upper and lower limbs and cardiovascular system loose their normal function in all the daily life activities. Lifting is evaluated with the isokinetic Liftask ${ }^{*}[3,13]$. With another analytic, functional, psychological and socio-professional data, the deconditioning syndrome is well documented and gives way for new treatment $[1,4,8,9,10]$.

Isokinetic exercises are integrated in our specific treatment: the spine functional restoration program; thus, the deconditioning syndrome is reversible if a multidisciplinary team is in charge of this program $[17,18]$. It integrates a physical rehabilitation with a multimodal pain management. This active back rehabilitation, without any passive usual techniques, is the key for returning to work for the whole population.

At the outcome of the programs end ant 2 years after, $80 \%$ of patients return back to work or ready to prospect for a job and stay active [15]. Earlier the patient returns to work, better is the result. This method is a new way to approach preventive concepts.

\section{References}

[1] R.J. Gatchel, T.G. Mayer, P. Capra, P. Diamond and J. Barnett Quantification of lumbar function, part 6: the use of psychological measures in guiding physical functional restoration, Spine 11 (1986), 36-42.

[2] M. Herlant, J. Vanvelcenaher and H. Delahaye, Evaluation isocinétique du déficit musculaire chez les lombalgiques. Revue de Médecine Orthopédique 28 (1992), 2-3.

[3] N.D. Kishino, T.G. Mayer, R.J. Gatchel, M. McCrate Parrish, C. Anderson, L. Gustin and V. Mooney, Quantification of lumbar function, part 4: Isometric and isokinetic lifting simulation device in normal subjects and low back dysfunction patients, Spine 10 (1985), 921-927. 
[4] P. Masse, P. Voisin and M. Herlant, Mesure par inclinométrie électronique de la mobilité du rachis lombaire dans le plan sagittal, De Sèze S, Debeyre J, Held J.P. La rééducation et la spondylolisthésis lombaire, Expansion Scientifique Française, Paris, 1999, pp. 253-260.

[5] T.G. Mayer, S.S. Smith, J. Keeley and V. Mooney, Quantification of lumbar function. Part 2: Sagittal plane trunk strength in chronic low-back pain patients, Spine 10 (1985), 765-772.

[6] T.G. Mayer, Orthopaedic conservative care. The functional restoration approach, Failed spine surgery, Hanley and Belfus, Philadelphia, 1986, pp. 139-147.

[7] T.G. Mayer, R.J. Gatchel, N.D. Kishino, J. Keeley, H. Mayer, P. Capra and V. Mooney, A prospective short term study of chronic low-back pain patients utilising novel objective functional measurement, Pain 25 (1986), 52-68.

[8] T.G. Mayer, D. Barnes, N.D. Kishino, G. Nichols, R.J. Gatchel, H. Mayer and V. Mooney, Progressive Isoinertial Lifting Evaluation, part I: A standardised protocol and normative database, Spine 13 (1988), 993-997.

[9] T.G. Mayer, D. Barnes, N.D. Kishino, G. Nichols, K. Coval, B. Piel, D. Hoshino and R.J. Gatchel, Progressive Isoinertial Lifting Evaluation. Part II: A comparison with isokinetic lifting in a disabled chronic low back pain industrial population, Spine 13 (1998), 998-1002.

[10] T.G. Mayer, R.J. Gatchel, D. Barnes, H. Mayer and V. Mooney, Progressive Isoinertial Lifting Evaluation (erratum notice), Spine 13 (1988), 5.

[11] T.G. Mayer and R.J. Gatchel, Objective measurement of functional performance. Functional Restoration for Spinal Disorders: The Sports Medicine Approach, Léa and Febiger, Philadelphia, 1988, pp. 161-169.

[12] T.G. Mayer, V. Mooney and R.J. Gatchel, Contemporary Conservative care for painful spinal disorders, Léa and Febiger, Philadelphia, 1991.

[13] K.E. Timm, Isokinetic lifting simulation: a normative data study, J.O.S.P.T. 5 (1988), 156-166.

[14] J.L. Vanhee, P. Voisin, Vezirian and J. Vanvelcenaher, Isokinetic trunk flexors and extensors performance with and without gravity correction, Isokinetics and Exercise Science 6 (1996), 89-94.

[15] J. Vanvelcenaher, D. Raevel, G. O'miel, P. Voisin, P. Struk, T. Weissland, E. Aernoudts, P. Bibre, M. Goethals and P. Masse, Programme de Restauration Fonctionnelle du Rachis dans les lombalgies chroniques, Encycl Méd Chir Kinésithérapie - Médecine Physique - Réadaptation 26 (1999), 294 B10.

[16] J. Vanvelcenaher, P. Voisin, P. Struk, E. Divay, M. Goethals, E. Aernoudts, et al. Programme de Restauration Fonctionnelle du Rachis (R.F.R.) dans le cadre des lombalgies chroniques. Nouvelle approche thérapeutique (1re partie: Données du problème - Evaluation), Ann Réadaptation Méd Phys 37 (1994), 317-321.

[17] J. Vanvelcenaher, P. Voisin, P. Struk, E. Divay, M. Goethals, E. Aernoudts, et al. Programme de Restauration Fonctionnelle du Rachis (R.F.R.) dans le cadre des lombalgies chroniques. Nouvelle approche thérapeutique (2è partie: traitement - résultats - discussion), Ann Réadaptation Méd Phys 37 (1994), 323-333.

[18] P. Voisin, J. Vanvelcenaher, J.L. Vanhee, P. Bibre, E. Divay, P. Struk, Programme de Restauration Fonctionnelle du Rachis (R.F.R.) pour une prise en charge active des lombalgiques chroniques, Ann Kinésithér 21 (1994), 337-350. 


\title{
Low Back Problems and Return to Work: Isokinetics?
}

\author{
Regi Melcherts \\ OCA Institute, Amersfoort, The Netherlands
}

\begin{abstract}
About one third of the medical and social costs in the Netherlands are caused by disorders of musculoskeletal system. Especially Low Back Pain is one of the main causes for disability in the working population. Because of lack of sufficient employees and the progressive incline in financial costs for the employer and the society, maintenance of working capacity is becoming one of the major issues in the Netherlands. A new development that changes health care and the social security system that started about one year ago. A challenge for the medical and paramedical field to contribute to the society by participating in multi-disciplinary programs for Return to work. On the other hand the increasing pressure of insurance companies, the government and employers forces the medical and paramedical professional to show the results of their programs.

Questions about interactions between the pathology, the functional status and the conditions at work, all related to a successful Return to Work, require multiple investigations and measurements. Return-towork programs for the Low Back Pain patient consist of therapy and training for functional restoration, behavioural therapy and education for self-efficacy and managing the workload. Sometimes adjustments of the facilities at work are necessary. But most of all, the commitment of the employee and the employer are necessary to be successful.

To show the results of the Return to Work-programs not only questionnaires, but also measurements of the functional capacity offer opportunities. Although the validity of isokinetics is questionable for multijoint movements and functional tasks, isokinetics contribute in a specific way according our experience to evaluate a part of the functional restoration of the Low Back Pain Patient. Future research is necessary to determine the interest and necessity of isokinetics in Return-to-Work programs for the Dutch situation.
\end{abstract}




\title{
Isokinetic Assessment in School Children
}

\author{
Stefano Negrini, G. Pinochi, R. Carabalona, S. Della Foglia and P. Sibilla \\ Rehabilitation Department, Don Gnocchi Foundation, Care \& Research Institute, Milan, Italy
}

Studying muscular strength of the trunk could be an important goal in relation to back pain developed in teenagers. During the Italian Backpack Study we collected data about trunk strength during isokinetic exercise in school children. The aim of this paper is to present the normative parameters collected and the differences observed between males and females; flexion and extension; velocities used.

Population: $\quad 62$ school children (29 females); age 11.9 years $(10.7-12.11)$; weight $45.03 \pm 9,9$ $\mathrm{kg}$; height $149,2 \pm 7,08 \mathrm{~cm}$.

Instrument: $\quad$ Cybex NORM dynamometer with TEF modular component.

Movement: $\quad$ flexion/extension of the trunk.

Range of Movement: from $15^{\circ}$ in extension to $45^{\circ}$ in flexion.

Velocities:

90 and $60 \%$ sec. ROM and velocities were chosen according our experience to reduce any possible risk in this population.

Statistical analysis: Newman-Keuls and $\chi^{2}$ test. Body Mass Index normalised data with respect to subject's size, dividing the value of each parameter. Significance was set at $p=0.05$.

Peak and Mean Torque and Best Work (flexors and extensors) and Total Work and Mean Power (extensors) were different between sexes. At different velocities we found differences in all parameters. Between the performance of flexor and extensor muscles we found differences mainly at $60^{\circ} / \mathrm{sec}$ (Peak and Mean Torque, Peak Torque Angle, Acceleration Time, Best and Total Work), but also at $90 \% \mathrm{sec}$ (Peak Torque Angle).

It was possible to determine normative parameters in the age group studied. The age when differences in muscular performance appear between males and females is not uniquely described. According to our results, statistically significant differences appear in 11-year-olds, although not in all parameters tested. As expected, the performance is always different at different velocities. Differences between flexor and extensor muscles are greater at reduced velocity. 


\title{
Isokinetics and EMG: Standardised Measurement for the Lower Extremity
}

\author{
Peter Konrad, Birgit Schulte-Frei, Jürgen Freiwald \\ KinLab-University of Dortmund, Dortmund, Germany
}

Electromyography is a well-established method for detecting the neuromuscular activation of muscles during static and dynamic tasks. When combined with isokinetic dynamometers it provides a better understanding of how the total output torque is generated by the involved muscle groups. In our clinic the most commonly used activity is the knee - extension/flexion in an open kinetic chain. Activity of the lower extremity muscles such as the rectus femoris, medial/lateral vastus, the hamstring, gastrocnemius, tibialis and peroneus can be detected with surface EMG. A meaningful interpretation, however, requires that the movements be executed in a standardised fashion.

Isokinetic testing procedures provide ideal conditions for standardisation since the only uncontrolled parameter - torque - is directly and quantitatively measured and can be used to calculate EMG input/torque - output relationships.

EMG measurements are automatically synchronised with the torque and angle data. The bipolar raw EMG signal is rectified smoothed (root mean square) and averaged in time normalised cycles. MVC(maximum voluntary contraction)-normalised EMG signal is required for quantitative comparisons of inter-individual muscle patterns and to estimate neuromuscular exhaustion. In clinical settings the MVC normalisation is typically not feasible and the analysis thus focuses on more qualitative aspects of the averaged activation pattern:

- Does the muscle activity increase or decrease as a function of change in mechanical load.

- How do the agonist/antagonist muscles interact during extension and flexion.

Yet another class of analysis quantifies the time dependent changes of EMG within a set of repetitions and evaluates recruitment and fatigue phenomenon. For example, an increasing EMG/torque ratio indicates fatigue-related recruitment, which provides valuable information on the efficiency of the chosen strength training method for the selected muscle groups.

It is beneficial to complement isokinetic tests with other functional test such as manual muscle function tests, gait and bilateral squats. 


\title{
Maximal and Sub-Maximal Torque-Velocity Relationships for Human Knee and Ankle Extensor Muscles
}

\author{
Alf Thorstensson \\ Department of Sport and Health Sciences, Stockholm University College of Physical Education \\ and Sports, and Department of Neuroscience, Karolinska Institute, Stockholm, Sweden
}

There is a difference in speed dependence of muscle force/torque in vitro (electrically stimulated muscle preparations) and in situ (maximal voluntary knee extensor actions). In the latter, eccentric torque does not show the expected increase above the isometric level.

Comparing voluntary and electrically induced maximal and sub-maximal isokinetic knee and ankle extensor actions further elucidates this phenomenon here. 30 healthy male subjects performed unilateral, isometric, concentric and eccentric muscle actions. Custom-built isokinetic dynamometers were used for torque recordings, the Spark machine for the knee and PlanDor for the ankle. Knee extensors were tested in sitting and ankle extensors in standing on all four to isolate the action of the soleus muscle. Angle specific torque at $60^{\circ}$ (knee) and $90^{\circ}$ (ankle) was used for analysis. Speeds ranged 10-360\% $/ \mathrm{sec}$ for the knee and $5-30 \%$ sec for the ankle. Electrical stimulation was applied percutaneously to torque levels corresponding to 30 (knee and ankle), 50, 70 and 90\% (knee) of maximal voluntary torque. Sub-maximal constant voluntary activation of soleus was performed with direct visual feedback of soleus EMG at a level corresponding to $30 \%$ of maximum voluntary torque. The torque-velocity relationship for maximal voluntary actions showed similar features for knee and ankle extensors, i.e. eccentric torque remaining at the isometric level and concentric showing a gradual decrease. Interestingly, also voluntary sub-maximal eccentric ankle extensor torque was on the isometric level. Electrically stimulated eccentric torque, on the other hand, exceeded the isometric by 37\% (ankle) and 9-31\% (knee). These findings from electrical stimulation support the notion that a tension regulation mechanism is present during eccentric maximal voluntary knee and ankle extensor actions. The sub-maximal voluntary ankle extensor data indicate that this regulation may be related to the action type as such rather than to its higher force potential. 


\title{
The Use of Isokinetics in Treatment of Patellofemoral Problems
}

\author{
Urs Geiger, P. de Leur, M. Göring and P. Coste \\ GYM medico, Basel, Switzerland
}

The Swiss Association of Extensive Physiotherapy (SGEP) shows in this presentation the role of isokinetics in the first Swiss protocol for treatment of patellofemoral pain syndrome (PFPS).

Based on study of literature, own experiences and other protocols, we describe the possibilities of isokinetics in training, evaluation and testing. It is important to mention, that in the SGEP-Protocol also other methods for testing, evaluation and documentation like for instance EMG and functional force measurements are integrated. Other therapeutic interventions, like manual therapy, massage and electrotherapy can be part of the treatment, are however not fixed.

The use of isokinetics in testing and evaluation gives us several important patellofemoral-specific parameters like:

- Peak and average force moments of the quadriceps in relationship to bodyweight

- The existence of force-inhibition (break-phenomenon) or small amplitude oscillations in eccentric movement - the location and sector of the break

- The H/Q-Ratio

- Involved/uninvolved differences (left/right)

In our presentation we pronounce the studies and publications, which mentions the importance of this specific parameters.

Based on several other publications we conclude that by using isokinetics we have possibilities to create optimal training input and minimal pain by using the following principles:

- Regarding the results of bio-mechanical studies in choosing specific angles to reduce patellofemoral joint reaction forces

- Choosing higher velocity for decreasing the compression forces, level of pain and simultaneously influencing different muscular qualities

- Choosing isolated concentric activity in the beginning of the rehabilitation.

- Limiting the ROM for pain reduction but using the overflow reactions in velocity and angle.

The use of isokinetics for testing and evaluating we can create a criterion-based rehabilitation within time-based principles. In this way the next step in the protocol does not only depend on physiological time factors or common medical decisions.

At last we should pronounce that the construction of this protocol gives us possibilities to evaluate the therapeutic and rehabilitation interventions in the protocol and to evaluate the protocol itself. 

Poster Abstracts 



\title{
Isokinetic Assessment of Ankle Muscle Strength after Surgical Repair of the Achilles Tendon
}

\author{
E. Brassinne ${ }^{1}$, R. Leclerc ${ }^{2}$, D. Mouraux ${ }^{1}$, P.-M. Dugailly ${ }^{1}$ and Y. Andrianne ${ }^{3}$ \\ ${ }^{1}$ Department of Physical Therapy and Rehabilitation, C.U.B., Erasmus Hospital, Belgium \\ ${ }^{2}$ Department of Physical Therapy and Rehabilitation, Vesale Hospital, Belgium \\ ${ }^{3}$ Department of Orthopedics and Traumatology, C.U.B., Erasmus Hospital, Belgium
}

The results, only based on clinical criteria, are excellent in 80 to $90 \%$ of surgical repairs of the Achilles tendon in the literature. The absence of objective assessment of the strength of the ankle prompted us to measure isokinetic parameters of the ankle joint after surgery.

A population of 18 patients is studied after surgical suture or reconstruction of Achilles tendon ruptures. The length of the follow-up is 1 to 6 years. A Cybex $340 \AA$ isokinetic dynamometer is used to test the dorsal and the plantar flexion of the ankle joint in two positions, prone (extended knee) and supine (flexed knee), at the speeds of $30 \%$ sec. and $120 \%$ sec., with three repetitions. The parameters "peak torque" and the "work" are taken into account because of their sensitivity. Total work during a tiredness test (twenty repetitions) is also studied in plantar flexion.

We don't observe any significant difference for the isokinetic parameters in dorsal flexion. In plantar flexion, all the differences between parameters of healthy and operated side are statistically significant, ranging between $11 \%$ to $24 \%$. The greatest loss is observed for the supine test at $120 \%$ sec.: $24 \%$ $(\mathrm{p}<0.001)$ for the peak torque and $23 \%(\mathrm{p}<0.001)$ for the work. During the tiredness test a decrease of the total work of $23 \%$ is noted $(\mathrm{p}<0.001)$. 


\title{
Isokinetic Concentric and Eccentric Evaluation of the Ankle Flexors and Extensors
}

\author{
M. Claes, V. Longue, R. Leclercq and C. Gregoir \\ UERI, CHU A Vésale, route de Gozée, 6110 Montigny le Tilleul, Belgium
}

\section{Introduction:}

The aim of this study was to provide descriptive eccentric and concentric strength of ankle's flexors and extensors, comparing dominant and non-dominant side.

\section{Methods:}

20 young healthy non-athletic adults (10 women and 10 men), mean aged 22.5 years old were tested on a CYBEX 6000 dynamometer.

The position was prone with $0^{\circ}$-knee extension. The range of motion was $-20^{\circ}$ of dorsiflexion (DF) to $50^{\circ}$ of plantar flexion (PF). Speeds were $30,120,150^{\circ}$. S-1 for concentric mode and $30,60,90^{\circ}$. S-1 for eccentric mode.

\section{Results:}

In women, PF's peak torque was from $46 \mathrm{Nm}$ to $78 \mathrm{Nm}$ on concentric mode, and from $79 \mathrm{Nm}$ to 91 $\mathrm{Nm}$ on eccentric mode. DF's peak torque was respectively from $9.1 \mathrm{Nm}$ to $15 \mathrm{Nm}$ and $23 \mathrm{Nm}$ to $27 \mathrm{Nm}$.

In men, PF's peak torque was from $62 \mathrm{Nm}$ to $107 \mathrm{Nm}$ on concentric mode and from $112 \mathrm{Nm}$ to 129 $\mathrm{Nm}$ on eccentric mode. DF's peak torque was respectively from $8.2 \mathrm{Nm}$ to $21 \mathrm{Nm}$ and $35 \mathrm{Nm}$ to $38 \mathrm{Nm}$.

No difference was observed in peak torque between dominant and non-dominant side.

$\mathrm{DF} / \mathrm{PF}$ ratio was mean 0.15 on concentric mode and 0.3 on eccentric mode. The ratio is not modified by neither speed, gender or side tested.

Peak torque was correlated with body weight only for the plantar flexion in both genders.

\section{Conclusion:}

Plantar flexors are stronger than dorsiflexors. No difference is found between dominant and nondominant leg. 


\title{
Isokinetic Assessment and High Level Sports
}

\author{
T. Cotte and J.M. Ferret \\ Centre de Médecine du Sport de Lyon Gerland, Lyon, France
}

\section{Introduction:}

Today, high level sports practices required high athletic capacity. The aim of this study is to check the different parameters of strength (maximal force, maximal power and strength at high speed) for high level athletes coming for 5 different kinds of sports to improve training process.

\section{Methods:}

5 disc throwers, 12 acrobatic skiers, 5 running sprinters, 20 soccer players, 7 Alpine skiers have been tested using an isokinetic dynamometer (Cybex Norm) at different speed for flexors and knee extensors in seated position. Several parameters have been measured:

1. Peak torque at each speed.

2. Peak torque/body weight.

3. Flexors/extensors ratio for each speed.

4. Eccentric flexors peak torque/concentric extensors peak torque ratio.

5. Eccentric peak torque/concentric peak torque ratio for the same muscular group.

\section{Results:}

1. There is no significant bilateral unbalance whatever the parameter studied and whatever the speciality.

2. From one sport to another one, the values of these parameters are significantly different.

\section{Conclusion:}

1. There is no significant bilateral unbalance, even for asymmetrical sports as soccer.

2. This result point out significant characteristics related to the sport specificity.

3. We suggest using these results on the practical field for management of athletic training and for injury prevention for sports people who benefit of isokinetic testing. 


\title{
Strength Training and Isokinetic Evaluation on Young Soccer Players
}

\author{
T. Cotte ${ }^{1}$, J.C. Chatard ${ }^{2}$ and J.M. Ferret ${ }^{1}$ \\ ${ }^{1}$ Centre de Médecine du Sport de Lyon Gerland, Lyon, France \\ ${ }^{2}$ Laboratoire de Physiologie, GIP Exercice, CHU de Saint Etienne, St-Etienne Cedex 2, France
}

\section{Introduction:}

In soccer, explosive efforts i.e. sprints, jumps, kicking and tackles are often the starting point of decisive actions. The question whether or not strength training could be a better means than soccer itself to improve muscle strength and maximal running speed without any risk for young players remains (Archambault et al. 1995, Trolle et al. 1993).

\section{Methods:}

24 young soccer players ( $17 \mathrm{yr}$. old) have been divided into a strength training $(\mathrm{ST}, \mathrm{n}=12)$ and a control group $(\mathrm{CG}, \mathrm{n}=12)$. ST trained 2 times per week, 3 weeks during in a succession of heavy load strength training with technical soccer sets.

Training effect was evaluated on:

- Knee flexors and extensors peak torque with an isokinetic dynamometer (Cybex Norm) at different speed (from -60 to $500^{\circ} \mathrm{s}^{-1}$ ).

- Vertical jump measured with an Ergojump.

- Ball velocity with radar after a maximal soccer kick.

- Running speed over 10, 20,30 and $40 \mathrm{~m}$.

- Peak power on a treadmill ergometer developed to stimulate a $4 \mathrm{~s}$ sprint run.

- Number of injury.

\section{Results:}

- The strength training programme increased the peak power measured on the treadmill ergometer $(15 \%, \mathrm{p}<0,005)$ and the maximal running speed over $10 \mathrm{~m}(4,5 \%, \mathrm{p}<0,05)$, but not muscle strength.

- No injury was reported during for ST, while 6 injuries were reported for CG.

\section{Conclusion:}

A 3-week strength-training programme is a good means to improve speed and muscle power with no more risk of injury. 


\title{
Hamstring Muscle Strain: Interest of the Eccentric Assessment and Preventive Aspects
}

\author{
J.L. Croisier and J.M. Crielaard \\ Department of Physical Therapy and Rehabilitation, University of Liege, Belgium
}

Recurring muscle strains often hampers athletes. Therefore, the incidence of injured hamstrings becomes more elevated for track and field athletes, soccer and football players.

Twenty-three competitive athletes presenting a previous tear of the hamstring muscles were examined through concentric $\left(60\right.$ and $240 \%$ s) and eccentric (30 and $\left.120^{\circ} / \mathrm{s}\right)$ isokinetic testing.

Our results reflected the following:

- In reference to the non-involved side performances, the reduction of peak torque (PT) of the injured muscles reached an average of $10 \%$ during concentric actions and surpassed $20 \%$ in eccentric.

- The relationship between relative PT (PT by body weight) and angular velocity demonstrated significant differences in comparison with those of selected non-injured sprinters, most notably in the eccentric sector.

- Classical concentric hamstrings/quadriceps $(\mathrm{H} / \mathrm{Q})$ ratio did not distinguish the strained muscles of the normal contra-lateral thigh. A mixed ratio ( $\mathrm{H}$ eccentric/Q concentric) appeared significantly reduced $(0.75 \pm 0.23)$ for the injured leg in comparison with the contra-lateral non-injured limb

- $(0.90 \pm 0.16)$ and with a control population $(0.98 \pm 0.14)$.

- For the eccentric mode, the angle of maximal effectiveness (AME) of the strained muscles occurred in an abnormal articular sector corresponding to a weaker hamstring muscle stretch than normal.

The persistence of such abnormalities demonstrates the frequent shortcomings of current rehabilitation programmes that neglect the terminal phase of muscular eccentric strengthening, particularly in the muscle lengthening position. By another way, the frequent occurrence of muscular imbalances concerning contra-lateral muscles considered as healthy demonstrates the interest of a preventive application of isokinetic evaluations for athletes at substantially increased risk of hamstring muscle strain. 


\title{
Interest of a Specific Training to Reduce DOMS
}

\author{
J.L. Croisier, G. Camus and J.M. Crielaard \\ Department of Physical Therapy and Rehabilitation, University of Liege, Belgium
}

Strenuous or unaccustomed exercises frequently cause delayed onset muscle soreness (DOMS), especially when eccentric contractions are involved.

10 moderately active male volunteers were randomly assigned to two age-matched groups: a control group (CG; $n=5)$ and a trained group (TG; $n=5)$. All subjects were submitted to two isokinetic provocation sessions in the eccentric mode $\left(60^{\circ} / \mathrm{s}\right)$, consisting of three stages of 30 maximal contractions of the knee extensor and flexor muscles. The subjective intensity of DOMS were evaluated using a visual analogue scale before, immediately after, and 48, 72, $96 \mathrm{~h}$ after each eccentric session. Increased serum levels of creatine kinase (SCK) and myoglobin $(\mathrm{SMb})$ were used as indirect indices of exercise-induced muscle damage. The two isokinetic provocation sessions were separated by a period of 3 weeks during which the subjects of the CG abstained from strenuous exercise while the volunteers assigned to the TG were submitted to five training sessions (five stages of 10 sub-maximal eccentric contractions of the involved muscles).

The first provocation session was followed by severe muscle pain in previously active muscles and by significant increases in serum creatine kinase activity and myoglobin concentration in both groups $(\mathrm{p}<0.001)$; those reached peak values after $48 \mathrm{~h}$, with average levels superior to $35000 \mathrm{UI} / \mathrm{l}$ for SCK! While the mean values of DOMS, SCK and SMb remained practically unchanged over time after the second provocation session in the CG, training was accompanied by a significant $(\mathrm{p}<0.05)$ decrease of these variables (for example, mean SCK reached a value of only $716 \pm 274 \mathrm{UI} / 1$ ).

Assuming that postexercice DOMS and increased SCK-SMb values do indeed reflect exercise-induced damage to the muscle, the marked reduction of these variables after training lends strong support to the notion that specific training increases muscle resistance to the injury caused by eccentric contractions. These results are useful to implement training and rehabilitation programs including eccentric exercises. 


\title{
Isokinetic Evaluation of Hip Strength Muscle Groups in Unilateral Lower Limb Amputees
}

\author{
J.L. Croisier, B. Maertens de Noordhout, G. Camus, S. Hac, O. De Lamotte and J.M. Crielaard \\ Department of Physical Therapy and Rehabilitation, University of Liege, Belgium
}

The present study was designed to measure the isokinetic strength of the hip flexor-extensor and adductor-abductor muscle groups in unilateral lower limb amputees.

Thirty-three patients with unilateral lower limb amputation were included in this study. The subjects served as their own controls, the amputated side was compared to the intact side.

Using an isokinetic device, the peak torque, bilateral asymmetry and agonist/antagonist torque ratio were calculated for the hip flexor, extensor, adductor and abductor muscle groups.

A significant reduction of mean concentric peak torque measured on the amputated limbs was generally observed when compared to the intact contra-lateral limb $(\mathrm{P}<0.05)$. The mean percentage of decrease of peak torque was greater for the extensors than for the flexors $(33 \pm 20 \%$ and $22 \pm 23 \%$ respectively, at $30 \%$ angular velocity) and for the abductors than for the adductors $(23 \pm 22 \%$ and $11 \pm 19 \%$ respectively, at $30 \%$ angular velocity). Furthermore, the flexor/extensor torque ratio was significantly increased for the amputated limb $\left(1.25 \pm 0.33\right.$ versus $1.09 \pm 0.25$ for the intact limb at $\left.30^{\circ} / \mathrm{s}\right)$ The reduction of the abductor/adductor ratio of the operated limb reached the level of statistical significance at $60 \%$ s only $(1.26 \pm 0.46$ versus $1.07 \pm 0.36$ for the intact limb).

In conclusion, isokinetic testing revealed a significant reduction of the hip stabilising muscle strength as a consequence of lower limb amputation. The more marked decrease of the strength of the abductor and extensor muscle groups, resulting in imbalances of the agonist/antagonist ratios should be taken into account to improve the conventional rehabilitation programs. 


\title{
Isokinetic Hip Muscles Performance in Normal Subjects
}

\author{
P.M. Dugailly ${ }^{1,2}$, E. Brassinne ${ }^{1}$, D. Mouraux ${ }^{3}$, P. Remy ${ }^{3}$, V. Feipel ${ }^{3}$ and P. Klein ${ }^{2}$ \\ ${ }^{1}$ Physical Therapy Department, Erasmus Hospital, Université Libre de Bruxelles, Brussels, \\ Belgium \\ ${ }^{2}$ Research Unit on Manual Therapies, Erasmus Hospital, Université Libre de Bruxelles, \\ Brussels, Belgium \\ ${ }^{3}$ Department of Functional Anatomy School of Physiotherapy and Rehabilitation, University of \\ Brussels, Brussels, Belgium
}

Research involving isokinetic evaluation devices has mostly concentrated on function of the knee, shoulder, ankle and back. These evaluations have been used to assess muscular strength, muscular status, and progress during rehabilitation or agonist/antagonist balance. The aim of this paper is to determine isokinetic performance of six muscle hip groups (flexors/extensors, internal/external rotators, abductors/adductors).

Twenty-eight a-symptomatic students $(11 \mathrm{E}, 17 \Gamma)$, performed three consecutive isokinetic evaluations of both limbs at 60 and $120^{\circ} \mathrm{s}^{-1}$. They had no history of hip or back injury and were considered as amateur sportsmen.

Our findings indicate that males achieved significant higher peak torque's values at every speed. No differences were found between left and right sides. Peak torque's were significantly larger at $60^{\circ} \mathrm{s}^{-1}$, except for male adduction and female extension. Significant differences between agonist and antagonist muscle performance were also found with an exception at $120^{\circ} \mathrm{s}^{-1}$ for the male group. The agonist/antagonist ratios ranged from 0.74 to 1.48 were significantly influenced by velocity. They differed only between groups at $120^{\circ} \mathrm{s}^{-1}$.

We have confirmed the influence of velocity on isokinetic hip muscle performance and obtained higher values in comparison to the results of previous studies. This study revealed a hierarchy of hip muscle performance depending of velocity and gender. The ratios did not always show values similar to those mentioned in some previous studies. Functional evaluation using isokinetic testing should be routinely used to assess hip muscle performance in different pathologies because this parameter may be an indicator of pelvis instability. 


\title{
Isokinetic Measurement of Forearm Pronators Supinators and Wrist Flexors - Extensors in Normal Subjects
}

\author{
B. Forthomme, J.L. Croisier, C. Heinzius, N. Vrochidis, M. Foidart-Dessalle and J.M. Crielaard \\ Department of Physical Therapy and Rehabilitation, C.H.U. Sart Tilman, Liège, Belgium
}

The strength of the forearm pronator-supinator and wrist flexor-extensor muscle groups plays a prominent role in many professionals and sports activities.

Limited normative isokinetic strength data are available for these muscles. The aim of this study is to validate an assessment protocol and to establish normative values.

10 non-athletics ( 5 women and 5 men) mean aged 22 years old were selected for the forearm pronation and supination isokinetic evaluation.

10 other normal masculine subjects ( 23 years old on average) were selected for the wrist flexion and extension testing.

The protocol modalities included angular velocities of $30 \% \mathrm{~s}$ and $90 \% \mathrm{~s}$ in the concentric mode and $60 \% \mathrm{~s}$ in eccentric for all movements.

Each subject participated to a test-retest measurement. Reliability was excellent for peak torque values: the variation coefficients did not exceed $12 \%$ for the pro-supination data and $9.4 \%$ for flexion-extension.

Peak torque by body weight values $(\mathrm{N} \cdot \mathrm{m} / \mathrm{kg}$, means and standard deviations) are presented in Table 1 .

Table 1

\begin{tabular}{lccc}
\hline & CONC. $30^{\circ} / \mathrm{s}$ & CONC. $90 \% \mathrm{~s}$ & ECC. $60^{\circ} / \mathrm{s}$ \\
\hline Pronators & $0,14 \pm 0,03$ & $0,12 \pm 0,03$ & $0,15 \pm 0,03$ \\
Supinators & $0,12 \pm 0,03$ & $0,10 \pm 0,03$ & $0,16 \pm 0,04$ \\
Flexors & $0,32 \pm 0,05$ & $0,32 \pm 0,07$ & $0,39 \pm 0,08$ \\
Extensors & $0,16 \pm 0,03$ & $0,14 \pm 0,02$ & $0,23 \pm 0,07$ \\
\hline
\end{tabular}

For all movements involved, except wrist flexors in concentric trials, we observed a significant effect of angular velocity and mode of contraction.

The flexors/extensors ratios reached respectively $2,2.25,1.75$ for the conc. $30 \%$, conc. $90 \%$ s and ecc. $60 \%$ s modalities.

The pronators/supinators ratios values were all included between 1.08 and 1.14.

A dominant influence was detected only for the forearm supinators at high speed in the concentric mode $(\mathrm{p}<0.001)$ and wrist flexors at all conditions $(\mathrm{p} \leq 0.002)$.

In conclusion, we defined a reliable isokinetic strength measurement protocol for forearm prosupination and wrist flexion-extension and proposed preliminary normative data in normal subjects. 


\title{
Isokinetic Trunk Strength Evaluation in Chronic Low Back Pain Patients
}

\author{
C. Gregoir, R. Leclercq, M. Claes and S. Boulvin \\ UERI, CHU A. Vésale, route de gozée, 6110 Montigny le Tilleul, Belgium
}

\section{Introduction:}

The aim of our study is to evaluate CYBEX 6000 TMC testing in normal subjects and chronic low back pain patients.

\section{Material and methods:}

60 non athletic women and men mean aged 23 years old were selected as normal subjects and 56 men and 64 women mean aged 42 years old suffering of chronic low back pain as patients. All subjects were tested on concentric mode, within a range of motion of 50 degrees, at the speed of 30, 60, 90 and 120 degrees per second. Patients undergo a clinical and X-ray evaluation.

\section{Results:}

Normal values of flexors and extensors peak torque are closed to the published data. The flexors/extensors ratio is 0.69 in lower speed and 0.87 in higher speed. Peak torque is highly correlated to body weight in both gender and at any speed $(\mathrm{R}=0.68, \mathrm{p}<0.001)$; variation coefficient is lower when results are expressed normalised to body weight.

Group 2: important decrease of extensors peak torque is demonstrated in chronic low back pain patients $(\mathrm{p}<0.001)$. This predominant deficit in extensor muscles induces a reverse of flexors/extensors ratio. Strength measures are correlated to lumbar stiffness index. Reliability is excellent (0.98) in normal subjects. The learning effect is important in-patients.

To discriminate low back pain patients from normal subjects the peak torque of the extensors at high speed and the flexors/extensors ratio are the best parameters.

\section{Conclusion:}

Isokinetic testing of trunk flexors and extensors muscles is a safe, reliable and discriminating method to evaluate a functional impairment in chronic low back pain patients. 


\title{
Side Differences in the Strength of Knee Extensors And Flexors in Healthy Persons and Effect of Bal- ance Training
}

\author{
H.C. Heitkamp, F. Mayer, H. Horstmann, J. Weller and H.H. Dickhuth \\ Medical Clinic, Department Sports-medicine, University of Tübingen, Tübingen, Germany
}

Reports on differences in strength between right and left knee extensors and flexors showed a large variability. Usually one-sided strength training is implemented for compensation. We measured knee extensors and flexors in a group of 7 male and 8 female persons aged 31 and 24 years. Measurements were performed on an isokinetic system with $60^{\circ}$ for extension and $30^{\circ}$ for flexion. Within a period of 6 weeks 12 training units of balance training lasting $25 \mathrm{~min}$ each were performed on instability devices such as rolling board, mini trampoline and large rubber ball. Before training for extension females exposed $135 \pm 47 \mathrm{Nm}$ right and $133 \pm 38 \mathrm{Nm}$ left, however the individual difference being $14.9 \pm 10.0 \mathrm{Nm}$ and after training $154 \pm 48$ and $154 \pm 48 \mathrm{Nm}$ respectively, the difference was reduced to $0.63 \pm 0.74 \mathrm{Nm}$. The males reached $229 \pm 44$ right and $227 \pm 39 \mathrm{Nm}$ left, the individual difference being $22.4 \pm 16.6 \mathrm{Nm}$ which was reduced to $2.71 \pm 0.49 \mathrm{Nm}$ after a gain in strength to $252 \pm 44$ and $251 \pm 45 \mathrm{Nm}$, respectively. The measurements for the flexors before training showed for the females $75 \pm 17$ right and $73 \pm 18 \mathrm{Nm}$ for the left with an individual difference of $7.50 \pm 5.55 \mathrm{Nm}$, which was reduced to $1.38 \pm 1.06 \mathrm{Nm}$ after a gain in strength to $85 \pm 19$ and $85 \pm 18 \mathrm{Nm}$. The males set off at $129 \pm 33$ right and $128 \pm 31 \mathrm{Nm}$ left with a difference of $8.43 \pm 7.49 \mathrm{Nm}$ which reduced to $2.71 \pm 3.45 \mathrm{Nm}$ after a gain in strength to $141 \pm 29$ and $141 \pm 26 \mathrm{Nm}$. With isokinetic measurements the discrepancy between right and left was shown to be $11 \%$ for female and $10 \%$ for males for the extensors and 10 and $7.7 \%$ for the flexors. The remarkable training effect on side balance might be important for persons engaged in sports and for elderly persons for injury prevention. 


\title{
Isokinetic Evaluation of Knee Extensors and Flexors in Patients with Unilateral Symptomatic Gonarthrosis
}

\author{
Y. Henrotin ${ }^{1,2}$, J.L. Croisier ${ }^{2}$, R. Dumont ${ }^{2}$, O. Debry ${ }^{2}$, J.P. Huskin ${ }^{3}$, J.E. Dubuc ${ }^{4}$, J.P. Leflot ${ }^{4}$, \\ J.Y. Reginster ${ }^{2}$ and J.M. Crielaard ${ }^{2}$ \\ Bone and Cartilage Metabolism Research Unit \\ ${ }^{1}$ Médecine Physique et Revalidation Fonctionnelle, \\ ${ }^{2}$ Orthopaedic Surgery \\ ${ }^{3}$ University of Liège, Belgium \\ ${ }^{4}$ Hopital Princesse Paola, Marche-en-Famenne, Belgium
}

\begin{abstract}
Many papers have reported the relationship between knee osteoarthritis and weakness of the quadriceps. Furthermore, some studies suggested that quadriceps weakness is a primary risk factor for knee disability and progression of joint damage in persons with osteoarthritis of the knee. In this multicentric study, we measured the asymmetry in flexors and extensors performances in patients with unilateral symptomatic gonarthrosis. Relationships between muscle weakness, algofunctional status and radiological changes were also studied.

The isokinetic muscle assessment of knee extensors and flexors was performed in 20 patients with unilateral gonarthrosis (10 women and 10 men). The average age of the tested patients was 53 years old (range 38-68). All patients had symptoms of pain and stiffness of the knee, radiological signs of knee gonarthrosis and/or cartilage lesions evaluated by arthroscopy. Concentric muscle performances expressed as peak torque $(\mathrm{Nm})$, maximal work $(\mathrm{J})$ and maximal power $(\mathrm{W})$ were assessed by isokinetic dynamometers (Kin-trex 1000 and Cybex Norm) and recorded at the velocities of 60 and $240 \%$ sec. To obtain comparable data on peak torque, maximal work and power, the range of motion was assessed using a window of 100 degrees. Flexors/Extensors ratios were also calculated. Asymmetry (deficit) for all isokinetic parameters was calculated and correlated with the algofunctional status (Lequesne index) of the patients and the severity of the radiological lesions (kellgreen and Lawrence score).

Isokinetic torque of the quadriceps at $60 \% \mathrm{sec}$ and $240 \% \mathrm{sec}$ was found to be reduced by $22 \%$ and $18 \%$ respectively compared with the healthy one. By contrast, flexors strength was significantly reduced at $60 \% \mathrm{sec}$ but not at $240 \% \mathrm{sec}$. The same conclusions can be drawn with maximal work and power. Flexor weakness was significantly correlated with Lequesne index at $60 \% \mathrm{sec}(\mathrm{r}=0.70 ; \mathrm{p}=0.0006)$ and $240 \% \mathrm{sec}$ $(\mathrm{r}=0.74 ; \mathrm{p}=0.0005)$. On the other hand, no significant relationship between extensor weakness at $60 \% \mathrm{sec}$ and lequesne index was observed. Radiological score was not correlated with knee extensor and flexors weakness.

This study demonstrates that quadriceps performances are commonly reduced in patients with gonarthrosis whereas flexors are not systematically affected. On the other hand, flexors deficit is strongly correlated with the Lequesne index suggesting that flexors play also an important role in gonarthritic patient disability.
\end{abstract}




\title{
Isokinetic Strength and Endurance of the Lower Limp Musculature Ten Years after Achilles Tendon Repair
}

\author{
T. Horstmann, C. Lukas, F. Mayer, H.C. Heitkamp and H.H. Dickhuth \\ Department of Sports Medicine, University of Tübingen, Tübingen, Germany
}

Achilles tendon repair with following immobilisation causes atrophy of the calf musculature. Aim of the study was the long-term evaluation of the strength and strength endurance deficit ten years after the injury.

63 patients (48 male, 15 female) were tested in the eccentric $\left(60^{\circ} / \mathrm{s}\right)$, isometric and concentric $(60 \%$ s, $180^{\circ} / \mathrm{s}$ ) mode for plantar- and dorsiflexion with a Lido Active dynamometer 10.8 years after Achilles tendon rupture repair and a 6-week immobilisation.

The mean peak torque of the 3 best of 5 movements and the work over 1 minute $\left(180^{\circ} / \mathrm{s}\right)$ were evaluated for plantar- and dorsiflexion. The circumference of the calf musculature and the range of motion of the ankle (ROM) were measured. Differences to the non-operated side were calculated with the Wilcoxon matched pairs test and corrected with the Bonferroni method.

For the mean peak torque in all modes there was no difference between the operated and non-operated legs ten years after the immobilisation. The work over 1 minute was markedly reduced for the operated leg in plantarflexion (516 J operated, $634 \mathrm{~J}$ non-operated, $\mathrm{p}<0.001$ ). Also the circumference of the calf musculature $(-1.1 \mathrm{~cm})$ and the $\operatorname{ROM}\left(-3.5^{\circ}\right.$ plantarflexion, $-1.9^{\circ}$ dorsiflexion) were reduced $(\mathrm{p}<0.001)$.

A selective atrophy of the slow twitch fibres seems to be relevant for the durable loss of musculature and the weaker calf musculature in the strength endurance test. After reconstruction of the Achilles tendon the early remobilization should be chosen. At the end of the rehabilitation more strength endurance training must be done. 


\title{
Assessment of Elbow Extension after Functional Surgery on Tetraplegics
}

\author{
Brigitte Houssin, L. Bottero and J.F. Janin \\ Centre de Rééducation et de Gériatrie, Pontault-Combault, France
}

\section{Introduction:}

Tetraplegic people benefit more and more of functional surgery. Resuscitation of elbow extension allows improving significantly autonomy of these people. A specific protocol dedicated to the assessment of the elbow on tetraplegics is proposed using an isokinetic dynamometer CYBEX NORM ${ }^{\circledR}$.

\section{Methods:}

9 normal subjects and 16 tetraplegics who benefit of elbow resuscitation (uni- or bilateral) for a total of 27 surgered elbows (10 triceps deltoïd (TD) transfer, 17 biceps triceps (BT) transfer) have been tested.

Subjects were in a seated position with chest stabilization, arm and body have been strapped on the back seat of the machine to avoid compensation. The dynamometer has been adjusted in order that the motion (elbow extension/flexion) takes place in a sagital vertical plan.

Gravity correction has been managed using a counter balance weight fixed to the lever arm.

Tested ROM is $0.120^{\circ}$. Training on the machine consists in a few movements and a rest period just before a real test of 3 reps (extension/flexion) at $120 \%$ sec.

\section{Results:}

Peak torques registered during extension are very weak (15 Nm for TD transfer-11 Nm for BT) compared to normal subjects $(59 \mathrm{Nm})$. In flexion, the peak torque is lower with BT $(12 \mathrm{Nm})$ compared to TD $(34 \mathrm{Nm})$. Average value for normal subjects is $54 \mathrm{Nm}$.

\section{Discussion:}

Peak Torque in extension is weak compared to normal subjects. The weakness in flexion after BT confirms litterature datas. Using an isokinetic dynamometer is a good tool to imagine multicentric tetraplegic elbow testing in order to evaluate surgeries and rehab protocols and to set a follow up of those elbows surgered. 


\title{
Influence of a Lumbar Support Belt on the Isokinetic Strength Performance of Trunk Flexors/Extensors: Healthy versus CLBP Subjects
}

\author{
K. Kerkour \\ Chief-physiotherapist, Hospital of Delémont, Delémont, Switzerland
}

\section{Introduction:}

The lumbar supports are often used to alleviate low back pain. The aim of this study was to determine the following of the isokinetic measurements of trunk flexion and extension performance with lumbar support and the measurements without support in healthy subjects and those who have a history of recurring Low back Pain (LBP).

\section{Methods:}

Fifty-five healthy subjects ( 32 men, 20 women) and thirty-two CLBP subjects (19 men, 13 women) were assigned to perform maximal isokinetic force, in a sagittal plane, of the extensors and trunk flexors (6 trials) with and without a lumbar support belt. We used a Lido Isokinetic device, at the speed of 60 and $120 \%$ s. The subjects were in a "seat-standing" position and the centre of the dynamometer axis was between L4/L5. The range of motion was of sixty grades.

\section{Results:}

Healthy subjects: There was no statistically difference in the strength performance with or without a lumbar support belt in a-symptomatic subjects.

LBP subjects: In the recurring low back pain population, however, strength performance of the trunk extensors increased of about $20 \%(\mathrm{P}<0.001)$ with a lumbar support belt, while the trunk flexors were not influenced by the brace.

Ratio Flexors/Extensors: With a lumbar support belt the ratio flexors/extensors has improved. It is between " $0.8-0.9$ ".

\section{Conclusion:}

A lumbar support belt has no influence on the recruitment of trunk muscles in healthy subjects; while in a CLBP population we have an improvement in the recruitment of trunk extensor muscles. The ratio flexors/extensors tends to be as the ratio of healthy populations. 


\title{
Isokinetic Evaluation of Flexor/Extensor Trunk Muscles: Healthy versus CLBP subjects
}

\author{
K. Kerkour
}

Chief-physiotherapist, Hospital of Delémont, Delémont, Switzerland

\section{Introduction:}

The aim of this study is to compare if we have a difference in different parameters of isokinetic strength (peak torque, ratio and in total work) at two speeds $(60 \%$ s and $120 \%$ ).

\section{Methods:}

Fifty-five healthy subjects ( 32 men, 20 women) and thirty-two CLBP subjects (19 men, 13 women) were assigned to perform maximal isokinetic force of the extensors and trunk flexors (6 trials) with a Lido Isokinetic device, at the speed of 60 and $120 \%$ s. The subjects were in a "seat-standing" position and the centre of the dynamometer axis was between L4/L5. The range of motion was of sixty grades.

\section{Results:}

Extensors: at the two speeds the CLBP subjects have an important loss of peak torque: about $40 \%$ $(\mathrm{P}<0.001)$ comparatively with healthy subjects. This loss is identically for the two sexes.

At $60 \%$ s the total work of the CLBP subjects is inferior of about $45 \%$ comparatively with healthy subjects. This difference is sensibly more important at $120 \%$ s: about $50 \%(\mathrm{P}<0.0001)$. There was no sex difference.

Flexors: The loss of force is less than extensors, it is about $20 \%$ at $60 \% \mathrm{~s}(\mathrm{P}<0.001)$ and about $25 \%$ at $120 \% \mathrm{~s}(\mathrm{P}<0.001)$. This loss is sensibly the same for the two sexes.

The total work loss is about $30 \%(\mathrm{P}<0.0001)$ for the two sexes and speeds.

Ratio Flexors/Extensors: The CLPB ratio is different from the one of a healthy subject. In a healthy population the ratio is comprise between " $0.7-0.8$ " while in a CLBP population this ratio is about " 1 ".

\section{Conclusion:}

The CLBP subjects have a loss of force and endurance of trunk's extensors muscles comparatively with healthy subjects. This diminution of extensors force and endurance disturbs the flexors/extensors ratio. Our results show the importance of using an intensive rehabilitation program to restore the force of the trunk's extensors muscles and to improve their endurance. 


\title{
Eccentric Re-education as Treatment of Chronic Lateral Epicondylitis
}

\author{
R. Leclercq, P. de Sa, M. Claes, S. Boulvin and C.Gregoir \\ UERI CHU A. Vésale, Route de Gozée, 6110 Montigny le Tilleul, Belgium
}

\section{Introduction:}

The aim of this open study is to determine the feasibility and efficacy of pro-supination eccentric reeducation in chronic lateral epicondylitis.

\section{Material and Methods:}

22 patients mean aged 42 years old with ultrasonography proven lateral epicondylitis were trained on a CYBEX 6000 dynamometer. 10 training's of 6 X 15 repetitions at the speed of 30 and $90 \%$ sec were conducted twice weekly after a concentric and eccentric evaluation. At the beginning the resistance was limited at $50 \%$ of the peak torque. The mean duration of symptoms was 8.8 months and all the patients had at least two previous treatments (NSAID, physiotherapy, and steroid injection). Pain (VCS), Thomsen test, grip strength, ultrasonography and isokinetic parameters were studied.

\section{Results:}

Pain, Thomsen and ultrasonography thickening decreased after treatment, while grip strength improved. $82 \%$ of the patients were improved; on global assessment the improvement is considered as good or very good in $65 \%$. In 6 patients the ultrasonography became normal.

The first isokinetic evaluation showed a decreased pronation/supination ratio compared to contralateral normal arm. After re-education there was no modification of isokinetic parameters whatever the clinical result was.

\section{Conclusion:}

More than two third of the patients were clinically improved by pro-supination eccentric re-education. 


\title{
Isokinetic Trunk Strengthening in Chronic Low Back Pain Patients
}

\author{
R. Leclercq, M. Claes and C.Gregoir
}

UERI, C.H.U.A. Vésale, 6110 Montigny-le-Tilleul, Belgium

\section{Introduction:}

The aim of this study is to determine the efficacy of isokinetic muscle reinforcement in the treatment of chronic low back pain.

First study concerned 80 patients, 44 women and 36 men, mean aged of 42 years old who have been trained on CYBEX 6000 TMC isokinetic dynamometer.

The mean duration of symptoms was 23 months and all patients had at least two previous treatments.

Re-education consisted on mean 14 training by active concentric trunk extension and flexion strengthening.

An isokinetic trunk strength evaluation and a functional capacity questionnaire were done before and after treatment.

\section{Results:}

Flexors peak torque only improved at high speed; extensors peak torque highly significantly improved at all speed $(\mathrm{p}<0.001)$. Flexors/extensors ratio decreased significantly but did not reach normal values at high speed. $71 \%$ of patients improved their functional capacity.

Comparing a group of patients treated by classic physiotherapy, flexors and extensors peak torque improved but the ratio was not modified.

\section{Conclusion:}

More than $70 \%$ of chronic low back pain patients improved subjectively and objectively with isokinetic muscle strengthening. Isokinetic but not "classical" re-education improved the flexors/extensors ratio. 


\title{
The Quantification of Reciprocal Strength Relations in the Shoulder at Different Movement Velocities
}

\author{
F. Mayer, I. Krauss, T. Horstmann, P. Janssen, H.C. Heitkamp and H.H. Dickhuth \\ Medicine Clinic, Department of Sports Medicine, Clinic of Orthopedic Surgery, Eberhard-Karls \\ University of Tübingen, Tübingen, Germany
}

The shoulder is functionally a ball-joint whereby high mobility is coupled with rather low stability. Muscular balance appears to be decisive for dynamic stability, since the passive stabilisers act only at the end of the extent of the movement in order not to limit the joint mobility. Altered strength relations are frequently described as "muscular dysbalances or deficits" and are considered to be cause of various shoulder pathologies, whereby objective quantification is difficult. The influence of the movement velocity on the strength relations is presently not clarified.

The definition of uninfluenced muscular strength relations (peak torque quotient of reciprocal muscle groups) was made on a total of 166 untrained men (NM) and 83 untrained woman (NW). Determination of the strength relation or reciprocal muscle groups (quotients of the maximum torques) was made for extension/ flexion $(\mathrm{Ex} / \mathrm{Fl})$, abduction/adduction $(\mathrm{Ab} / \mathrm{Ad})$ and rotation movements (ERO/IRO). A further differentiation was made by various working modes and movement velocities (isometric, concentric: $60 \%$ s, $180 \%$ s, eccentric: $60^{\circ} / \mathrm{s}, 120 \%$ s).

The results show a decreasing quotient of reciprocal strength relations in the sequence: $\mathrm{Ab} / \mathrm{Ad}>\mathrm{Flex} / \mathrm{Ex}>\mathrm{Aro} / \mathrm{Iro}$. In spite of clearly differing absolute values [Nm] there was no statistically relevant difference in strength relationship in dependence on the working mode (isometric, concentric, eccentric) and tested velocities ( $p>0.05)$. The only remarkable finding was a slightly reduced strength relation of rotation movements under concentric stress.

It is concluded that, no relevant velocity dependence can be assumed in calculating the reciprocal strength relations. A different (neuro) muscular structure of the muscle groups involved with a corresponding effect on the velocity of strength performance thus does not appear to exist or could not be measured. The methodical background with various influence factors (positioning, fixation) on the reliability and validity must be discussed. 


\title{
The Effect of Sub-Maximal Eccentric Isokinetic Training on Strength and Cross Sectional Area of the Human Achilles Tendon
}

\author{
D. Mouraux ${ }^{1}$, B. Stallenberg ${ }^{2}$, A. Moury ${ }^{1}$, P-M. Dugailly ${ }^{1}$ and E. Brassinne ${ }^{1}$ \\ ${ }^{1}$ Department of Physical Therapy and Rehabilitation, Erasmus Hospital, Université Libre de \\ Bruxelles, Brussels, Belgium \\ ${ }^{2}$ Department of Radiology, Erasmus Hospital, Université Libre de Bruxelles, Brussels, Belgium
}

This study was designed to investigate the effects of six weeks sub-maximal eccentric training on isokinetic concentric and eccentric peak torque and cross sectional area (CSA) (muscle and tendon) of gastrocnemius.

Six men and four women $(24,7 \pm 3,2$ years $)$ volunteered as subjects for this study.

Peak torque value for plantar flexion were determined at $30 \% \mathrm{~s}, 60 \% \mathrm{~s}$ and $90 \% \mathrm{~s}$ (concentric and eccentric) using a Cybex Norm before the training, 3 weeks after the beginning and at the sixth week.

The CSA values of the gastrocnemius were measured at $2 \mathrm{~cm}$ up the insertion on the calcaneum and at mid-calf before and after training.

The training protocol was based on the works of Stanish used on the chronic tendinitis. This was effected unilaterally (dominant leg) on an isokinetic dynamometer.

After six weeks, peak torque was increased $(\mathrm{p}<0.001)$ in all tests concentric and eccentric for both legs (trained and untrained). The CSA results were not significantly increased.

The results of this study suggests that after 6 weeks of sub-maximal eccentric training: strength training effects are not completely specific to the contraction type used in training, a contra-lateral transfer is present and neither muscle nor tendon hypertrophy was observed suggesting that neural adaptation contributes to strength increase. 


\title{
Evaluation of the Velocity-Dependent Increase of Passive Resistance in Spastic Plantar Flexors
}

\author{
G. Rabita ${ }^{1}$, L. Dupont ${ }^{2}$, A. Thévenon ${ }^{3}$, G. Lensel-Corbeil ${ }^{1}$, C. Pérot ${ }^{4}$ and J. Vanvelcenaher ${ }^{2}$ \\ ${ }^{1}$ Laboratoire d'Etudes de la Motricité Humaine, Faculté des Sciences du Sport, Université de \\ Lille 2, France \\ ${ }^{2}$ Centre de Rééducation et de Réadaptation Fonctionnelles Spécialisées L'Espoir, Lille- \\ Hellemmes, France \\ ${ }^{3}$ Service de Médecine Physique et de Réadaptation, CHRU de Lille, France \\ ${ }^{4}$ UMR CNRS 6600, Biomécanique et Génie Biomédical, Université de Technologie de Com- \\ piègne, France
}

\section{Introduction:}

Spasticity was defined as "...a motor disorder characterised by velocity-dependent increase in tonic stretch reflex..." Several authors, however, had demonstrated the contribution of muscular mechanical changes to the increased resistance to stretch in spastic muscles. The aim of this study was to evaluate quantitatively the resistance to stretch with respect to the velocity of passive dorsiflexion.

\section{Methods:}

The resistive torque obtained at $0^{\circ}$ dorsiflexion was measured during passive dorsiflexions imposed at various velocities ranged from 10 to $300 \%$ s using a Cybex ${ }^{\circledR}$ Norm ${ }^{\text {TM }}$ dynamometer on 11 spastic (aged $47 \pm 20$ years) and 9 control (aged $47 \pm 20$ years) subjects. During the passive movements, surface EMG was recorded from the medial and the lateral gastrocnemius, the soleus and the tibialis anterior.

\section{Results:}

With regard to the raw resistive torque, no statistical difference was observed between the control and the spastic groups. On the contrary, normalised with respect to the values obtained at $10 \%$, the resistive torque was statistically higher in the spastic group for velocities ranging from 120 to $300 \%$ s. Surface EMG analysis did not reveal any electromyographical activity whatever the condition and the group tested.

\section{Discussion:}

This study shows that, whatever the stretch velocity applied at the ankle, the raw resistance was not higher in the spastic compared with the normal subjects. On the other hand, the increase of this resistance with velocity, represented by the normalised values, was higher in spastic subjects. This velocitydependence without muscle activation could confirm the impaired viscoelastic behaviour of spastic plantar flexors. 


\title{
Clinical Muscle Testing and Isokinetic Evaluation of Latissimus Dorsi and Donor Site
}

\author{
P.Rémy ${ }^{1}$, P. Putzeys ${ }^{2}$, V.Theuwissen ${ }^{1}$, D. Mouraux ${ }^{1}$, F.Schuind ${ }^{2}$, Y.Andrianne ${ }^{2}$ \\ ${ }^{1}$ Department of Kinesiology, Erasme, Brussels, Belgium \\ ${ }^{2}$ Department of Orthopedies and Traumatology, Erasme, Brussels, Belgium
}

\section{Introduction:}

Transfer of the vascularized latissimus dorsi muscle has been widely used to cover soft tissue defects after trauma. However, neither the log-term effect of this procedure on the global function of the shoulder, nor the ability of the remaining muscle to compensate the lost function of the latissimus dorsi (adduction, internal rotation and extension of the shoulder) has been precisely studied. The aim of the present study was to assess the clinical shoulder function and the balance of the shoulder stabilising muscles by instrumented muscle testing.

\section{Results:}

All patients rated the appearance of the donor extremity, as good and no subject perceived any functional impairment at the donor site. The mean Constant's functional score was 96 for the operated shoulder and 98 on the opposite site. Isokinetic evaluation showed that adduction torque of the operate shoulder, at a speed of 60 degrees per second, was weaker. The difference between both shoulders was significant $(p=0.020$ and $p=01014)$, The difference in total work and average power at 60 degrees/second for adduction was significantly different ( $p=0.42$ and $p=0.023$ respectively). The value for internal rotation were significantly different only for the mean value of peak torque at 120 degrees/second $(p=0.038)$.

\section{Conclusion:}

The data of this study showed that removal of the latissimus dorsi muscle had no effect on Constant's functional score. However, adduction strength was significantly weaker on the donor site. Internal rotation strength was affected only at 120 degrees per second. 